\title{
Against Laplacian Reduction of Newtonian Mass to Spatiotemporal Quantities
}

\author{
Niels C.M. Martens
}

Received: 20 June 2017 / Accepted: 22 February 2018

This is a post-peer-review, pre-copyedit version of an article published in Foundations of Physics. The final authenticated version is available online at: http: //dx. doi. org/10. 1007/s10701-018-0149-0

\begin{abstract}
Laplace wondered about the minimal choice of initial variables and parameters corresponding to a well-posed initial value problem. Discussions of Laplace's problem in the literature have focused on choosing between spatiotemporal variables relative to absolute space (i.e. substantivalism) or merely relative to other material bodies (i.e. relationalism) and between absolute masses (i.e. absolutism) or merely mass ratios (i.e. comparativism). This paper extends these discussions of Laplace's problem, in the context of Newtonian Gravity, by asking whether mass needs to be included in the initial state at all, or whether a purely spatiotemporal initial state suffices. It is argued that mass indeed needs to be included; removing mass from the initial state drastically reduces the predictive and explanatory power of Newtonian Gravity.
\end{abstract}

Keywords Mass $\cdot$ Newtonian gravity $\cdot$ Reduction $\cdot$ Laplace $\cdot$ Mach

I would like to thank Tushar Menon, Tom Møller-Nielsen, Oliver Pooley, Carina Prunkl and David Wallace for useful discussions and comments on earlier drafts of this paper. I am grateful for questions and comments from the audiences at "Foundations 2016" at LSE, London, and the Ockham Society at the University of Oxford. This material is based upon work supported by the Arts \& Humanities Research Council of the UK, a Scatcherd European Scholarship, and in part by the DFG Research Unit "The Epistemology of the Large Hadron Collider" (grant FOR 2063). The major part of this paper was written while I was at Magdalen College and the Department of Philosophy, University of Oxford, including a twomonth research visit to Princeton University (supported by the AHRC Research Training Support Scheme and a Santander Academic Travel Award).

N.C.M. Martens

Institute for Theoretical Particle Physics and Cosmology, RWTH Aachen, Germany

Tel.: +492418027060

E-mail: martens@physik.rwth-aachen.de

ORCID: 0000-0002-2839-1387 


\section{Introduction \& Motivation}

Pierre Laplace had a problem. A big one. He wanted to know what information, if any, would be sufficient to predict the future. This question was asked in a much more precise way by Henri Poincaré [1,2]. What is the minimal choice, if any, of initial variables and parameters that corresponds to a well-posed initial value problem - that is, for any combination of the associated initial determinate values, the relevant laws will determine a unique evolution [3]?

Poincaré was the first to explicitly link this problem to the substantivalismrelationalism debate about space and motion $[1,4]$. In retrospect, in the context of theories of gravity - in this paper we will focus on Newtonian Gravity (NG) - we have now been focusing for more than three centuries on the question of whether the initial data need include absolute or merely relational quantities. That is, do we need positions, velocities and/or accelerations relative to absolute space (i.e. substantivalism), or is it sufficient to consider distances, velocities and accelerations relative to other material bodies (i.e. relationalism)?

Given the age and the importance of this debate, it should be surprising that we have only very recently started considering explicitly whether we need the absolute scales of dimensionful quantities (i.e. absolutism) or merely the ratios between them (i.e. comparativism). Do we require an absolute distance scale, or only ratios between distances? Do we require an absolute mass scale, or only ratios between masses? In fact, mass has been the central example in these recent discussions [5-7] - more on this in Subsection 3.1.

A third aspect of Laplace's problem has been left out entirely: need we include in our initial conditions, besides spatiotemporal quantities, any notion of mass at all? Or can mass - the absolute mass scale and/or the mass ratiosbe reduced to initial spatiotemporal quantities (e.g. accelerations), such that a purely spatiotemporal initial state can still correspond to a well-posed initial value problem, rendering such choice of purely spatiotemporal variables a candidate solution to Laplace's problem?

In other words, this papers considers Laplacian reductionism about mass as it features in Newtonian Gravity. I will not distinguish between gravitational and inertial mass, but take them to be equivalent - at least in the sense of being numerically proportional. I am interested in reducing mass within $\mathrm{NG}$, rather than reducing $\mathrm{NG}$ as a whole to a more fundamental theory and identifying a counterpart of Newtonian mass in that reduced theory. I will argue that Newtonian mass is not reducible, in these specific Laplacian and intra-theoretic senses, to spatiotemporal quantities; we should be primitivists (i.e. non-reductionists).

I take the wish to solve Laplace's problem - or rather a slightly extended version of it, as discussed in Section 2-as sufficient motivation for considering Laplacian reductionism about mass, but it is worth briefly listing other possible motivations. One modest motivation, which is arguably implicit in Laplace's problem, would be the desideratum of metaphysical parsimony, i.e. Ockham's razor. If a specific concept does no work - in some sense to be further 
specified below - at all, Ockham urges us to expunge it from our theory. This is to be distinguished from the much more radical motivation that drives many empiricists: the urge to get rid of all unobservables/ theoretical terms, regardless of any other virtues they might have, such as explanatory power. This is what motivates the different project of Machian reductionism-reconstructing the masses from spatiotemporal data not just at the initial time but at any number of instants of time, as described in detail below. Machian reductionism is of roughly equal historical importance as the Laplacian project, but much more broad and radical and therefore importantly distinct from it. It will become important later on that it is merely the more modest motivation - a methodological principle that I believe everyone should apply, regardless of any sympathies for empiricism - that drives the specific Laplacian project of this paper. One may but need not sign up to the more radical (Machian empiricist) motivation to pursue the Laplacian project (and in fact I do not).

Finally, three more specific reasons for thinking, or at least hoping, that Laplacian reductionism may be true, are to be mentioned briefly. Firstly, we will see in Subsection 4.2 that Machian reductionism is in fact true (to a certain extent). This naturally leads us to wonder whether this result extends to the much more restricted Laplacian context.

Secondly, an extremely succesful method of accounting for observed galactic rotation curves goes under the name of MOdified Newtonian Dynamics (MOND) [8]. Newton's law of Universal Gravitation gets enhanced, but only in the regime where the accelerations are much smaller than $a_{0} \equiv 10^{-10} \mathrm{~m} / \mathrm{s}^{2}$. In the context of MOND, it thus seems much more natural to consider initial conditions in terms of accelerations. Now, proving Laplacian reductionism about mass (to accelerations) within NG would not thereby prove it for MOND, but since MOND is equal to NG for accelerations much larger than $a_{0}$, a refutation of reductionism within NG (in that regime) has as a corollary that mass cannot be so reduced within MOND either.

Thirdly, I have argued elsewhere [7,9] that Baker's attempt [6] at defending comparativism about mass (see the second aspect of Laplace's problem above) in fact boils down to a Laplacian reduction of mass to accelerations.

\section{The Project}

Why did we ever introduce mass in the first place? We cannot observe (a particle having the property of having) mass as directly as, for instance, the location of a massive object (relative to us). What we do observe however-pace Barbour [2] - is that some trajectories (i.e. relative locations over time) do occur in nature, say two celestial bodies approaching each other at ever increasing speed, and other trajectories never occur, say two celestial bodies executing 
the Argentine tango ${ }^{1,2}$. We postulate the primitive notion of mass because it explains why certain patterns are allowed by nature and other patterns are not. Mass therefore becomes indirectly observable. (Or so the standard story goes. It is the aim of this paper to explore whether the same explanatory power can be obtained without primitive masses.) More specifically, if we include the values of the primitive masses in the initial state of our models of NG, and postulate laws that refer to this notion of mass, it turns out that we can find unique solutions to the corresponding initial value problems and thereby fix the evolution of the system up to infinity. Including mass in our theory thus allows us to 1) predict future states of the world based on past data. But not only that. It turns out that in addition we can 2) explain the observed particle trajectories. Why this is the case is most easily illustrated by showing why the reductionist theory is lacking in this respect, as will be done in several places below. In a nutshell, in the mass theory (i.e. the primitivist theory) the initial variables and parameters can take on all $^{3}$ possible values, whereas the reductionist theory exhibits ad-hoc, holistic, brute (i.e. unexplained!) constraints on the initial values (which cannot even be formulated without piggy-backing on the mass theory). Finally, if we range over all the values the initial variables and parameters could take, and solve the initial value problems for each of these cases, we obtain the correct ${ }^{4,5}$ set of empirically possible models. In other words, including mass gives us the correct 3 ) counterfactuals and other modal claims of the Newtonian Theory of Gravity. What more could we want from a physical theory?

The three virtues above provide a rough characterisation of the 'work' that the notion of mass seems to do within our theory despite not being directly observable (or more precisely, as directly observable as relative distances). If we can show that spatiotemporal quantities could do that work all by themselves, then it was never really (i.e. fundamentally) the notion of mass which did all that work (contrary to the standard story above), and we could get rid of a primitive notion of mass and perhaps should do so on grounds of Ockham's

\footnotetext{
1 One might respond that we could simply hold a massive object in each hand, and feel the potentially different weights, despite there being no spatiotemporal difference. However, the experience of the strength of that force in one's brain is correlated with and reducible to the depth to which the massive object protrudes into our skin, that is our somatosensory nervous system. Even weighing an object in one's hand is consistent with the claim that all observation is spatiotemporal.

2 Similar sentiments are echoed by Bell ("[I]n physics the only observations we must consider are position observations..." [10, p.166]), Holland ("[A]ll experiments in the real world ultimately reduce to the determination of position. As far as we are aware this [assumption] is completely in accord with actual laboratory practice" [11, p.350]) and Jammer (" $[\mathrm{I}] \mathrm{n}$ the last analysis all measurements in physics are kinematic [i.e. spatiotemporal] in nature..." $[12$, p.6]).

3 Except perhaps for negative mass values, but these can be made irrelevant if we take it as a fundamental feature of the gravitational law that it is attractive and thus only cares about the magnitudes of the masses. (See Jammer [12, Ch.4] for a historical overview of the search for negative (gravitational) masses.)

4 In the regime of applicability of the theory.

5 See fn.6.
} 
razor. More specifically, for our specific (i.e. Laplacian) reductionist project to succeed, we would need to purge mass from our initial state, leaving only spatiotemporal quantities, and have our laws refer only to those spatiotemporal quantities. If the corresponding initial value problems give the correct, unique solutions, we can 1) predict future data using past data that is purely spatiotemporal. Provided there are no unexplained constraints on the initial data, we can also 2) explain the observed or allowed particle trajectories. Finally, if we then range over all the possible initial spatiotemporal states, and again get the correct ${ }^{6}$ set of empirically possible models, the 3 ) counterfactuals and other modal claims are also recovered without having invoked the notion of mass.

The most obvious way to proceed with this Laplacian reductionist project seems to be to find an operational definition of mass in terms of spatiotemporal quantities. We can then directly substitute ${ }^{7}$ the notion of mass in the initial state and the laws with these spatiotemporal quantities, and our work is done. Mach's famous operational definition of mass immediately springs to mind. Indeed, we will shortly start our discussion with Mach. However, it is important to flag at this point once more that Mach's more radical, empiricist project is substantially different from the more specific Laplacian project just outlined, as will be discussed in more detail below. Thus, although it would be imprudent not to start off our discussion with Mach's famous operational definition, Mach exegesis is not the aim of this paper; as soon as our Laplacian project diverges from his, we will leave Mach behind. (In another paper Machian reductionism plays a more primary role in my discussion - albeit under the name of Humean or Regularity eliminativism [14].)

Before moving on we need to make more explicit what was already implicit in the previous story: how far do we want to go? One current strand of re-

\footnotetext{
6 Correct in (at least) the sense of being in agreement with folk science- "If I had dropped this cup, it would have fallen to the ground"; "Two celestial bodies cannot execute the Argentine tango"; "If the two particles described in Subsection 3.1 had (in)sufficient initial velocity, they would escape (collide)". Of course we have access to counterfactuals only indirectly: we first choose the best theory to represent and explain the actual world (within the relevant regime), and only then infer counterfactual claims by inspecting what evolution this theory would generate for counterfactual initial conditions. In a sense it thus seems circular to claim that Newtonian Gravity generates the correct set of empirically possible worlds (within the Newtonian regime), beyond the non-trivial claim that Newtonian Gravity is indeed the best theory in light of the empirical data in the actual world (within the Newtonian regime). It is logically possible and it would be interesting to consider a totally different Newtonian theory, without mass, that manages to represent and explain the actual world as well as or even better than Newtonian Gravity with primitive masses, whilst nevertheless having different modal consequences. I criticise a similarly revisionist option elsewhere [7, Ch.3\&4.4.2]. In this paper I am in the first instance interested in the less radical project of trying to remove a primitive notion of mass from standard Newtonian Gravity without revising that theory - that is, while maintaining the same modal consequences. We are considering reducing mass to other primitives within the same theory, not coming up with a distinct theory. Once this fails, a distinct project could then be to search for such a revisionary theory as just described, but that is outside the scope of this paper.

7 Zanstra [13] adopts a similar 'substitution approach' in the analogous debate on relationalism about space.
} 
search considers a moderate version of reducing mass. It considers taking some notion of mass to be fundamental, namely the mass determinates - i.e. it is a matter of fact whether two massive particles are equally massive or not-but aims to derive its further quantitative structure - ordering $^{8}$, metric ${ }^{9}$ and additive structure ${ }^{10}$ - from for instance the dynamics ${ }^{11}$. Both the Laplacian and the Machian project are interested in reducing mass altogether ${ }^{12}$, not merely its quantitative structure. Keeping in mind the desideratum of metaphysical parsimony, it is simply not clear what work the fundamental mass determinates are still supposed to do once their quantitative structure has already been reduced away.

As I have already dismissed a famous argument against reductionism by McKinsey, Sugar \& Suppes elsewhere [7, §6.2], Section 3 dives straight into Mach's operational definition of mass. Section 4 treats historical responses to Mach, culminating in my main argument against Laplacian reductionism about mass in Subsection 4.3. Section 5 responds to two loopholes in the argument, before the conclusion that Newtonian mass is not reducible - in the Laplacian and intra-theoretic senses - to spatiotemporal quantities without loss of explanatory and predictive power.

\section{Mach}

Mach is well-known for providing the first operational definition of inertial mass [18]. He vehemently opposed employing 'hidden' metaphysical notions such as mass in physics in order to explain observable phenomena. The task of physics is merely the "abstract quantitative expression of facts" [18, p.502] concerning the relations between observable phenomena. He defines inertial mass (relations) in terms of observable accelerations (or more correctly, acceleration relations) only, a feat that so inspired the logical empiricists.

Consider two particles, which are either alone in the universe or approximately dynamically isolated from any other matter. If $F_{12}$ is the force exerted on particle 1 by particle 2 , and $F_{21}$ the force exerted on particle 2 by particle 1 , then Newton's third law gives $F_{12}=-F_{21}$. But Newton's second law also gives $F_{12}=m_{1} a_{12}$ and $F_{21}=m_{2} a_{21}$, where $a_{12}$ denotes the acceleration of particle 1 due to particle 2 , and vice versa. Combining this to eliminate the

\footnotetext{
8 Whether a massive particle is less or more massive than another particle with a different mass determinate.

9 The ratio between the masses of two massive particles.

10 How the mass of one massive particle compares to the combined mass of two other massive particles.

11 Dees [15] advocates a position like this. Perry [16] considers a more varied range of reductionist projects, including the Machian project.

12 See also Esfeld \& Deckert's project [17] and my paper on regularity comparativism and regularity eliminativism about mass [14].
} 
(directly) unobservable notion of force, we obtain ${ }^{13}$ :

$$
\frac{m_{1}}{m_{2}}=-\frac{a_{21}}{a_{12}} .
$$

We have operationally defined the mass ratio of these two isolated particles, or simply "named" $[18$, p.266] their acceleration relations as Mach would put it.

Of course our actual universe does not consist merely of (subsystems of) two isolated particles. Perhaps we could manually approximately isolate such systems (in turns) on the surface of the Earth where we dwell - or, since we can never perfectly isolate any system from gravity, obtain the mass ratios via the limit of a series of better and better isolated two-particle subsystems - but this would not be an option when considering celestial objects [20]. It definitely will not be an option in the generic messy, crowded worlds that we consider in this paper ${ }^{14}$. This raises the important question of whether the previous procedure can be consistently generalised to a larger system of interacting particles. Two issues arise. Firstly, the operational definition depends on the component of the acceleration of a particle that is induced by a single other particle, whereas we only have empirical access to the total acceleration. In Subsection 4.1 we will consider whether these individual components can be retrieved from the total acceleration.

The second issue is prior to the first, since it arises even if we could (per impossibile, in general) isolate each pair of particles (in turns). Consider a universe with three or more particles. Step 1: take particle 1 and 2 away from the other matter, such that they form an effectively isolated subsystem. Obtain their mass ratio from the acceleration ratio via Mach's protocol. Step 2: repeat for particle 2 and 3 . What can we now expect if we repeat this for particle 1 and 3 ? Will it satisfy the following consistency check:

$$
\left(\frac{m_{1}}{m_{3}}\right)_{s_{3}}=\left(\frac{m_{1}}{m_{2}}\right)_{s_{1}} \cdot\left(\frac{m_{2}}{m_{3}}\right)_{s_{2}},
$$

\footnotetext{
13 Note that in general $\frac{a_{21}}{a_{12}}$ depends on the reference frame, although it will be constant across inertial reference frames. Mach's definition therefore also depends on the first law [12, p.15], which provides the notion of an inertial frame. However, operationalising the inertial frames brings with it its own problems. Pendse [19] proves that there exists an infinite set of special non-inertial frames such that observers at rest with respect to those frames obtain positive and constant values for the mass ratios via Mach's operational definition, which nevertheless differ from the corresponding values found in the inertial frames. Importantly, those observers will not be able to tell that they are not in an isolated, inertial frame. Note that these problems are irrelevant to the project in this paper: we take the initial spatiotemporal quantities with respect to some inertial frame to be given, and use them to attempt to calculate the emergent masses and the evolution of the system. The Machian project"the heuristic aspect" in Pendse's terminology [19, p.55] — on the other hand starts out with observed trajectories only, and needs to somehow reconstruct the inertial spatiotemporal quantities before one may derive the mass (ratios).

14 Here I sympathise with Barbour's warning not to be "misled by the special circumstances of our existence. ... Take a billion of particles and let them swarm in confusion - that is the reality of 'home' almost everywhere in the universe. The stars do seem to swarm... We must master celestial [determination of mass ratios] and not be content with the short cuts that can be taken on the Earth, for they hide the essence of the problem" [2, p.137-8].
} 
where $s_{i}$ indicates the instance of the operational procedure (i.e. step) used to determine that mass ratio? If absolute ${ }^{15}$ masses are primitive properties of the particles, this condition is satisfied as a matter of logical neccesity. In Mach's framework, this equation is satisfied only if

$$
\left(-\frac{a_{31}}{a_{13}}\right)_{s_{3}}=\left(-\frac{a_{21}}{a_{12}}\right)_{s_{1}} \cdot\left(-\frac{a_{32}}{a_{23}}\right)_{s_{2}} .
$$

But the accelerations which particle 1 and 2 induce in each other and which particle 2 and 3 induce in each other place no (logical) constraints on the accelerations which particle 1 and 3 induce in each other. Mach acknowledges this. For him it is just a brute empirical fact that it does not matter which particle we use as a standard to compare every other particle to; any standard will provide the same mass ratios. But this is just another way of saying that the reductionist assumes a highly mysterious and holistic fact without any explanation whatsoever. A fact that is trivially explained - as a matter of logical necessity! - if absolute masses are taken to be fundamental. We here encounter the first loss of explanatory power for the reductionists - something that might not bother the Machian but does bother the Laplacian.

If this empirical fact is nevertheless assumed, one can then proceed by choosing one of the particles as the standard unit of mass, say $1 \mathrm{~kg}$ or $1 \mathrm{lb}$, in order to fix all the other masses via the consistently determined mass ratios. (Note that knowing the absolute accelerations would not by itself help to fix the absolute masses.) Mass thus seems to have been reduced to acceleration relations.

\subsection{Mach \& Comparativism}

Before evaluating Mach's definition qua reductionist project, it is worthwhile pointing out that this definition also makes him a comparativist about mass (as opposed to an absolutist). Absolutism about mass is the view that mass ratios $^{16}$ between material bodies obtain in virtue of the absolute (i.e., roughly, intrinsic [7, §1.1.2] [21]) masses of those bodies [5,7]. Comparativism denies this. Comparativists then often (but not always [14]) take absolute masses to have no empirical meaning; they are merely a convention. Thus, since Mach operationally defines only mass ratios, if anything, and arrives at absolute quantities merely via a convention, this makes him a comparativist.

Is any justification given for stopping at this point and not continuing to provide a further operational definition of the absolute masses? Before responding to this question, it should be pointed out that the absolutist should rejoice in Mach's achievement, as far as it goes. The absolutist acknowledges mass ratios of course, and has never claimed that masses (either the absolute

\footnotetext{
15 For comparativism about mass - defined below - an analogous issue of consistency or transitivity arises [21] [7, Ch.5].

16 Mass relations, more generally. But I will follow Baker [6] in focusing on mass ratios.
} 
masses or mass ratios) are 'directly observable' (or more correctly, as directly observable as relative distances). If mass were 'directly observable' the whole debate between absolutism and comparativism would not exist in the first place - so the absolutists always admitted the need for a method of measuring those mass relations. And this can of course only be done via (more directly) observable, spatiotemporal notions, such as acceleration. Once we have this operational definition of mass ratios though, do we also need a further operational definition of the absolute mass scale?

Since I have argued elsewhere in detail that Newtonian mass is absolute, let me only briefly rehearse the core of that argument here $[6,7,9]$. Consider a Newtonian world with two equally massive particles a distance $r$ apart, with a relative positive initial velocity $v$ and zero angular momentum. How will this world evolve?

Whereas this description corresponds to a unique choice of initial variables and parameters for the comparativist, the absolutist will demand that more information is needed: this description is compatible with uncountably infinitely many intrinsic masses. And, she claims, this choice is important, because for some choices of intrinsic masses the particles will escape each other and for other choices they will collide/coincide ${ }^{17}$ - two evolutions that are obviously empirically distinct - depending on whether the following inequality is satisfied:

$$
v>v_{e}=\sqrt{\frac{2 G m}{r}} .
$$

It is clear from this inequality that the evolution depends on the initial absolute masses of the particles, over and above their mass ratios. (Besides, once the initial masses are fixed, the corresponding absolutist initial value problem has a unique solution: absolutism is deterministic ${ }^{18}$.) The comparativist initial state lacks the resources to distinguish between these two categories of evolutions, with indeterminism between empirically distinct evolutions as a result. Absolute masses are empirically relevant; adding absolute masses to the initial state beyond mass ratios increases the predictive power.

Could we supplement Mach's project with an operational definition of the absolute mass scale? It seems that attempting to do so would not violate the spirit of the original project. The main thrust of the Machian project was the reduction of mass. Mach incorrectly interpreted 'mass' to refer to mass ratios only. If we manage to additionally reduce the absolute mass scale, this would complete the original project of reducing mass (now correctly understood as both mass ratios and an absolute mass scale).

The obvious candidate for such an operational definition is exactly the escape velocity scenario that was used to prove the empirical relevance of

\footnotetext{
17 In the case of non-zero angular momentum, the set of solutions that features coinciding particles is of measure zero. In that case we may need to turn to the evolution of shapes/angles to empirically distinguish the models, rather than coincidence.

18 Modulo some well-known exotic counter-examples [22, 23].
} 
absolute masses in the first place. The escape velocity inequality ${ }^{19}$ can be reformulated in terms of spatiotemporal quantities only: $v^{2}>v_{e}^{2}=2 a r$. This suggests that the absolute mass scale could be defined in terms of some ratio of $r, v$ and $a^{20}$. Although this seems unproblematic for the case of two particles, we will see below (Section 5) that this does not in fact generalise to more particles.

\section{Beyond Mach}

\subsection{Generalising to more particles}

Let us now evaluate Mach's project qua reductionism. Pendse famously points out that Mach's definition depends crucially on the simple two-particle scenariowhich initially seemed like a mere pedagogical simplification-and does not generalise to any number of particles [25]. Mach's definition requires the separate contributions induced by every other particle to the acceleration of a specific particle, whereas we only have empirical access to the total acceleration of that particular particle. In systems with too many particles the total acceleration underdetermines the individual contributions. More specifically, Pendse argues that, if we use only acceleration relations at one instant, the mass-ratios are not uniquely determined for systems of more than four particles. Moreover, even if we consider acceleration relations at any number of instants, systems with more than seven particles will not give a unique set of mass ratios. I will briefly outline the first argument here [12].

Let $n$ be the number of particles. $\mathbf{a}_{k}$ is the observed, induced total acceleration of the $k$ th body at $t_{0}$, and $\hat{\mathbf{u}}_{k j}$ the unit vector in the direction from body $k$ to body $j$ at $t_{0}$. Then

$$
\mathbf{a}_{k}=\sum_{j=1}^{n} a_{k j} \hat{\mathbf{u}}_{k j}, \quad(k=1, \ldots, n)
$$

where we solve for $a_{k j}\left(a_{k k}=0\right)$, the $n(n-1)$ unknown coefficients in $3 n$ linear equations, which represent the induced acceleration on particle $k$ by particle $j$ at $t_{0}$. It is these coefficients that Mach needs to fix the mass ratios. They are uniquely determined only if their number does not exceed the number of equations, $n(n-1) \leq 3 n$, and this is not the case for systems with more than 4 particles. QED. The reader is referred to Pendse's paper for the proof concerning acceleration data at any number of instants.

\footnotetext{
19 This inequailty governs the special case where the mass ratio is one, but this could easily be generalised

20 We may call this a (spatiotemporally) local operational definition. In Ref. [14] I discuss an attempt at a global definition, namely a reduction of the absolute masses to the full $4 \mathrm{D}$ mosaic of particle trajectories (and perhaps their mass ratios). Dasgupta provides an example of an alternative global definition. He introduces a notion of plural grounding, and argues that the totality of kilogram facts is plurally grounded in the totality of mass ratios [24].
} 


\subsection{Including other spatiotemporal quantities}

Narlikar responds by echoing the thought that underlies the suggested operational definition of the absolute mass scale: accelerations might be insufficient, but we have other spatiotemporal notions at our disposal [26]. In particular, we can measure inter-particle distances as well as accelerations, and insert them into the Gravitational Law ${ }^{21}$. Setting Newton's constant to one for convenience, we get the following equation for the (arbitrarily $\operatorname{chosen}^{22}$ ) $\mathrm{x}$ component of the acceleration of particle 1 due to the gravitational interaction of all the other particles, at $t_{0}$ :

$$
a_{1, x}\left(t=t_{0}\right)=\frac{m_{2}\left(x_{2}-x_{1}\right)}{r_{12}^{3}}+\frac{m_{3}\left(x_{3}-x_{1}\right)}{r_{13}^{3}}+\ldots+\frac{m_{n}\left(x_{n}-x_{1}\right)}{r_{1 n}^{3}},
$$

where it is understood that the positions and distances are measured at $t=t_{0}$ also. These, together with $a_{1, x}$, can be observed, resulting in a linear equation of the form

$$
A_{12} m_{2}+A_{13} m_{3}+\ldots+A_{1 n} m_{n}=X_{1},
$$

where only the $m$ 's are unknown. Repeating this procedure for a total of $(n-1)$ different instants, we get $(n-1)$ (supposedly) ${ }^{23}$ linearly independent equations, allowing us to solve for $m_{2}, m_{3}, \ldots m_{n}$. Observing in addition a single acceleration-component of any of the other particles at $t=t_{0}$ only is sufficient to determine the remaining $m_{1}$.

\subsection{The main argument}

It is here that we diverge from Mach's reductionist project. Mach's project was of a reconstructive, descriptive and epistemological/empiricist nature. It is the project of humans reconstructing (after the fact!) the masses from the appearance of the four-dimensional mosaic generated by God ${ }^{24}$. Therefore,

\footnotetext{
21 Pendse [27] objects that we do not have independent empirical access to the Gravitational Law. However, in the context of the project in this paper we simply take the laws as given. In fact, Mach and Pendse's own projects take Newton's Laws as given, so why could Narlikar not add the Gravitational Law to this?

22 This awkward arbitrariness stems from the slightly surprising attempt to reduce the $n$ degrees of freedom of a scalar, mass, to the $3 n$ degrees of freedom of a vector, acceleration $[7, \S 6.6]$. This arbitrary choice of an $\mathrm{x}$-axis seems especially unjustified in the homogeneous Euclidean space in which Newtonian Gravity lives (pace Knox [28]) — we seem to have implicitly chosen a preferred axis, in a space which has no structure to ground such a notion. Of course, if mass is a primitive notion it is easy to show that nothing depends on the choice of axis; it is not clear why the same would necessarily be true in the reductionist framework. As argued elsewhere, removing this arbitrariness by using all components of acceleration instead forms no improvement $[7, \S 6.6]$.

23 Although these equations may be linearly independent in general, presumably not all specific instances will be so. What to do with those deviant cases? Perhaps it will turn out that these specific systems are of measure zero in the space of solutions, and that that gives us some reason to ignore them. Or perhaps these cases result in infinitely many solutions which are all empirically equivalent. Or perhaps choosing a different set of instants to measure the distances suffices to restore linear independence. All of this remains to be shown though.

24 See Ref. [14] for a discussion of reconstructing absolute masses using this approach.
} 
using spatiotemporal data at any number of instants is perfectly acceptable; we are here not in the business of explaining part of the data (the future data) from other parts of the data (the initial state). And this project had better work! We have been applying Newtonian physics succesfully for over three centuries now. We have modeled and predicted the behaviour of the planets in our solar system, based on presumed knowledge of the masses of those planets. Thus, there had better be some response to the potential problems with Narlikar's argument as elaborated upon in footnote 23, unless we want to invoke some error theory ${ }^{25}$ about the way we have been doing Newtonian physics for the past three centuries.

In this paper we are however interested in the much more specific, metaphysical, Laplacian project of explaining our actual world by deterministically generating it from the initial conditions. That is, we are 'playing God', rather than reconstructing some true, after-the-fact statements about God's creation. Hence, we are only allowed to use spatiotemporal data at the initial time ${ }^{26}$. The future data is part of the explanandum, not the explanans. Using it would be explanatorily circular. The tools used by Narlikar (and by Pendse when proving his second claim) are not available in the context of this project of solving Laplace's problem ${ }^{27}$. In a slogan: compared to Machian reductionism, Laplacian reductionism wants to achieve more (i.e. explanatory and predictive power) with less (i.e. spatiotemporal data at the initial time only).

Does this mean that the Laplacian reductionist project is doomed? No. We can retain Narlikar's insight - that we have more spatiotemporal data at our disposal than merely accelerations - but restrict ourselves to that additional data at the initial time only.

If we could find an operational definition of the masses in terms of the initial spatiotemporal notions, then this would guarantee that these initial spatiotemporal notions would suffice (via some law which is obtained by substituting all references to mass by its operational definition) to generate a unique evolution, since this is guaranteed by the initial masses (plus distances positions and relative velocities). As we have seen that initial accelerations are insufficient, we might follow Narlikar's lead by including distances and inserting them into the gravitational law. We start of with his Eq. 6 for the $\mathrm{x}$-component of the acceleration of particle 1 at $t_{0}$, but instead of supplementing it with similar equations at different instants, we consider the analogous equations for the other particles at the same instant. For instance:

$a_{2, x}\left(t=t_{0}\right)=\frac{m_{1}\left(x_{1}-x_{2}\right)}{r_{21}^{3}}+\frac{m_{3}\left(x_{3}-x_{2}\right)}{r_{23}^{3}}+\frac{m_{4}\left(x_{4}-x_{2}\right)}{r_{24}^{3}}+\ldots+\frac{m_{n}\left(x_{n}-x_{2}\right)}{r_{2 n}^{3}}$.

25 Beyond of course the obvious errors in the quantum and relativistic regimes.

26 Or perhaps an infinitesimally small initial period of time, see fn. 36 .

27 For similar reasons Schmidt's reduction of mass $[12,29]$ is disqualified. 
We obtain the matrix equation $G \mathbf{m}=\mathbf{a}$, where $G$ is the following $n \times n$ matrix:

$$
G=\left(\begin{array}{cccc}
0 & \alpha_{12} & \cdots & a_{1 n} \\
\alpha_{21} & 0 & & \vdots \\
\vdots & & \ddots & \vdots \\
\alpha_{n 1} & \cdots & \cdots & 0
\end{array}\right)
$$

where $\alpha_{i j}=\frac{x_{j}-x_{i}}{r_{i j}^{3}}$. Since $\alpha_{i j}=-\alpha_{j i}{ }^{28}, G$ is an antisymmetric matrix. But the determinant of an antisymmetric matrix with odd dimensions is singular! Recall that it is a property of the determinant that $|G|=\left|G^{T}\right|$ and $|-G|=$ $(-1)^{n}|G|$. For an antisymmetric matrix $\left(G^{T}=-G\right)$ these properties combine to give $|G|=\left|G^{T}\right|=|-G|=(-1)^{n}|G|$. For odd $n$ then $|G|=-|G|=0$. Since a unique solution requires a non-zero determinant, this proves that there is no unique solution of masses. QED.

\subsection{Unpacking the argument}

What exactly follows from this? If the determinant had been non-zero, then Laplacian reductionism would have been straightforwardly successful. It is less straightforward whether the vanishing of the determinant rules out such reductionism. A vanishing determinant (for systems with an odd number of particles) proves that either there are no solutions or there are infinitely many solutions. Given that standard Newtonian Gravity has some solutions, we know that there are at least some sets of initial spatiotemporal quantities that fall into the latter category. Are there any sets that fall into the former?

\section{Horn 1: No solutions}

One might think that the following set of initial spatiotemporal quantities does not correspond to any (physical) solution. Consider a simple example of a system consisting of three particles. Figure 1 depicts three collinear particles, with the middle particle being one meter away from each of the outer particles. The middle particle has zero acceleration, and the outer particles each an acceleration of $1 \mathrm{~m} / \mathrm{s}^{2}$ outwards. Since gravity is supposed to be attractive, one might think that there are no mass solutions corresponding to this scenario, but there are in fact two categories of (mathematical) solutions: one in which the middle

\footnotetext{
28 This is true only because Newtonian Gravity contains both Newton's third law and the principle of equivalence of gravitational and inertial mass - without for instance the latter Eq. 6 would not have been as simple. This seems to suggest that if we were to go beyond Newtonian Gravity by adding other forces which, for instance, do not obey a similar equivalence principle (such as the Coulomb Force), the argument against reductionism would collapse. This cannot be true however, since this would only introduce more unknowns (i.e. the electric charges) without extra 'knowns' to determine those unknowns (unless perhaps the additional force depended on velocity and we could measure the velocities to aid us).
} 

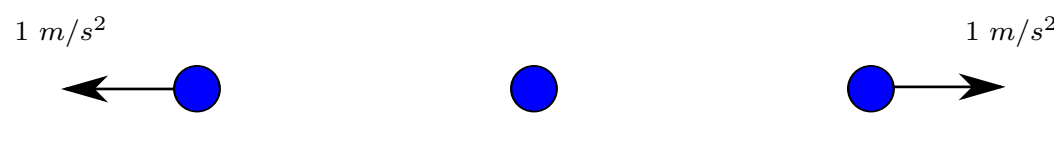

$1 m$

$1 m$

Fig. 1: First example of a set of accelerations to which no (positive) mass distribution corresponds.
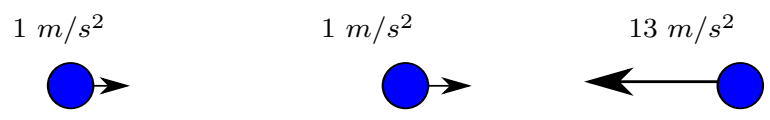

$1 m$

$1 m$

Fig. 2: Second example of a set of accelerations to which no mass distribution (either positive or negative) corresponds.

particle has a negative mass and the other two a positive mass, and vice versa. Although there is in fact a solution, this seems 'unphysical', since standard NG with mass includes the postulate that masses are always positive ${ }^{29}$. Standard NG thus does not contain these types of solutions. What should we do with such 'non-physical' solutions? Perhaps the reductionist could respond by claiming that we can somehow throw away these mathematical solutions since they are non-physical. We discuss such moves below. Instead I will now move on to a more decisive example, where there are not even any mathematical solutions.

In the second example all accelerations are 'inwards', which seems prima facie compatible with the attractive nature of gravity. In Figure 2 the two particles on the left accelerate with $1 \mathrm{~m} / \mathrm{s}^{2}$ to the right, and the third particle accelerates in the opposite direction with $13 \mathrm{~m} / \mathrm{s}^{2}$. It is easy to show that there is no solution in terms of masses, not even negative masses.

Could the reductionist just choose to (a priori) rule out those deviant sets of initial spatiotemporal quantities? Especially Humeans about laws of nature might be tempted by this approach. For instance, in the Mill-Ramsey-Lewis Best Systems approach $[30,31]$ any true statement that is part of the best system to axiomatise the data counts as a law. Thus, if the statement that rules out these deviant sets of initial spatiotemporal quantities is part of the best system, we could just postulate it as a law of our reductionist theory.

29 Or, that the gravitational law does not care about the sign of the masses. 
Compare this to the Humean solution to the problem of the arrow of time: if the Past Hypothesis (i.e. the claim that the initial entropy of the universe was sufficiently low [32]) forms part of the best system, this allows us to promote it to the status of law.

Apart from the standard complaints that such statements are not at all the type of beast that we normally consider as a candidate for law-hood, it is important that any such postulated constraint on the initial conditions is neither ad hoc, nor unexplained. Moreover, this constraint should be formulatable without (implicitly) referring to masses, that is without piggy-backing on the theory that takes masses to be primitive ${ }^{30}$. There are several reasons to believe that these conditions are not satisifed.

Whereas the restriction on entropy was straightforward - the initial entropy had to be below a certain value - the restrictions that would rule out the deviant set of initial spatiotemporal quantities - or more specifically initial accelerations - that do not correspond to any mass solution are much more complicated. In fact, no value of initial acceleration for any individual particle is ruled out from the start; the constraint takes on a holistic form instead. Only if a particle is located 'on the outside', do we all of a sudden require that its acceleration is not directed 'outwards'. (Notice that this also holds for systems with an even number of particles.) Similarly, once the initial accelerations of all but one particle have been chosen, this can restrict the allowed values of the acceleration of the 'final' particle (even if that particle was on the 'inside'). Leaving out the acceleration of a single particle from the initial conditions is not an option since even when we do include this piece of acceleration the mass solutions are already underdetermined in some cases (see below), nor would this solve the former problem regarding outward accelerations. The choices of the initial accelerations of a particle thus depend on the choices for the initial accelerations of the other particles. It is as if the laws determine, after the fact, in a holistic sense, which initial accelerations were allowed in the first place. Namely, exactly those that correspond to initial masses. Inference to the best explanation suggests that that is the case exactly because there are fundamental (initial) masses. There is no non-ad-hoc, reductionist explanation for ruling out the deviant sets of initial accelerations, especially not one that does not piggy-back on the concept of mass. In contrast, these constraints are trivially explained (by the attractive nature of gravity) if we do take masses to be primitive.

\section{Horn 2: Infinitely many solutions}

Let us turn to the sets of initial spatiotemporal quantities that correspond to an infinite set of solutions in terms of (initial) masses. These sets underdetermine the masses, and since different masses correspond, in general, to

\footnotetext{
30 Pooley [33, §5.4] discusses analogous issues (concerning Sklar's relationalist manoeuvre of adding primitive accelerations to the initial conditions) in the analogous substantivalismrelationalism debate about space. See also Arntzenius [34, §5.7] on piggy-back relationalism.
} 


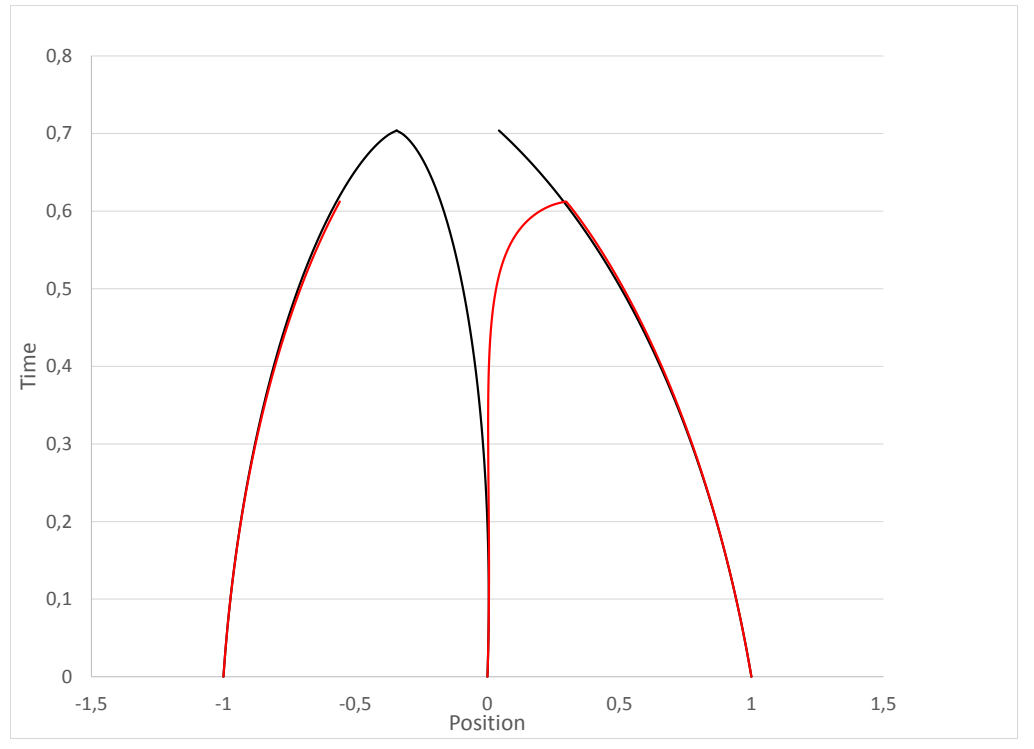

Fig. 3: The Smoking Gun: A numerical solution of the three-body problem in one dimension (black trajectories), superimposed on an alternative solution (red trajectories). Each three-body problem has only been solved until the first collision, as the theory breaks down at that point. The initial states of each set of three particles are identical with respect to the spatiotemporal quantities $\left(G=1, d_{12}=d_{23}=1, v_{1}=0.2, v_{2}=0.1, v_{3}=-0.5, a_{1}=1.25, a_{2}=-1\right.$, $\left.a_{3}=-1.5\right)$, but they differ in terms of their masses $\left(m_{1}^{r}=5.5, m_{2}^{r}=0.125\right.$, $m_{3}^{r}=4.5 ; m_{1}^{b}=m_{2}^{b}=1.2, m_{3}^{b}=0.2$ ). Note that they do not only differ in their absolute masses but also their mass ratios! These different sets of masses generate empirically distinct evolutions! Particle 2 collides first with particle 3 within the red solution, but first with particle 1 within the black solution.

different (metaphysical) evolutions of the system, an initial state that contains only spatiotemporal data leads to an indeterministic evolution (if there is any well-defined evolution in the first place). Such a reductionist theory will not provide the explanatory and predictive power that NG with primitive masses does.

The first, most obvious line of responses consists of variations on the theme that perhaps each set of infinite solutions is similar enough, in some sense, to 'count as one' and to therefore effectively form a single unique solution.

Variation 1: it might be the case that, even though each of these sets of initial spatiotemporal quantities corresponds to several distinct possible sets 
of masses each of which lead to metaphysically distinct evolutions, these respective distinct evolutions are in fact all empirically equivalent. If this is true for each set of initial spatiotemporal quantities that has multiple solutions, this would not only save the reductionist project, but also prove that the mass theory recognises distinct metaphysically possible models that are empirically indistinguishable, which violates the Principle of the Identity of Indiscernibles (that is, a specific instance of our principle of metaphysical parsimony, i.e. Ockham's razor).

Variation 2: Perhaps each set of infinitely many solutions consists of solutions that differ only with respect to the intrinsic masses but not with respect to the mass ratios. If so, the reductionist has at least partially succeeded by reducing the mass ratios, if not the absolute masses.

The easiest, most conclusive way to kill both variations with one stone is by providing a single counter-example to both. Figure 3 shows two superimposed numerical solutions of the three-body problem in one dimension. The solutions are generated from initial conditions that agree with respect to the spatiotemporal quantities, but disagree with respect to their initial masses (which in both cases are compatible with the spatiotemporal initial state) and moreover their mass ratios (against variation 2). Both solutions clearly generate empirically distinct evolutions (against variation 1), since in one case the middle particle collides first with the particle on the left, and in the other case its first collision is with the particle on the right ${ }^{31}$. Moreover, even if the mass ratios had been the same in this example, it would have served to reiterate the point made in Subsection 3.1 that absolute masses make an empirical difference. Thus, it would make salient that under variation 2 the need for fundamental absolute masses would remain, which anyway provide the mass ratios for free, thereby making such a partial reduction of the mass ratios good for nothing.

Secondly, the reductionist might suggest that including the ' $y$ ' and ' $z$ ' components of the acceleration might serve to remove the underdetermination and provide unique mass solutions. We should immediately feel uneasy about this suggestion: when attempting to fix $n$ mass degrees of freedom one would expect to need $n$ acceleration degrees of freedom, not an additional $2 n$ more! ${ }^{32}$ More on this in Section 5. But even when we do allow ourselves these extra degrees of freedom, this move will not work. The one-dimensional case is still a specific instance of the three-dimensional case. In scenarios were the ' $y$ ' and ' $z$ ' components of acceleration are zero, all components of acceleration together still underdetermine the masses and thereby the evolution of the system.

\footnotetext{
31 It might be argued that one cannot compare which particle is left or right of the middle between different solutions. One could avoid this by adding an extra particle sufficently far from these free particles to be dynamically isolated from them, in order to serve as a reference for, say, 'left'. However, the two solutions are clearly not each other's mirror image, so adding an extra reference particle is not really required.

32 On reflection, it is quite strange that we are trying to reduce the $n$ degrees of freedom of mass, a scalar, to acceleration, which - as a vector-has $3 n$ degrees of freedom, in the first place. This asymmetry provides the basis for an additional argument against reductionism developed elsewhere $[7, \S 6.6]$.
} 
Thirdly, the reductionist might bite the bullet and accept indeterminism (at the initial time only). Perhaps there are alternative, reductionist laws which allow for several possible evolutions of the initial spatiotemporal state - one for each of the evolutions that correspond to the mass solutions compatible with that initial spatiotemporal state - but once a specific evolution has 'begun' it follows through, deterministically, until the end. In other words, the laws are indeterministic relative to the initial instantaneous state, but not relative to an initial chunk of the evolution. At this point I can only respond by pointing out that the onus is on the reductionist to provide such indeterministic laws that generate the correct set of empirically possible evolutions ${ }^{33}$. Even if successful, it seems that such an approach would nevertheless weaken the predictive power of the theory.

\section{Bits and bobs}

Have we ruled out that mass can be reduced to initial spatiotemporal quantities? At least two issues need to be dealt with before we can conclude so.

The main argument rests on the substantive premise that the number of particles $n$ is odd. This may not be true of the actual world. Especially Humeans about laws of nature might jump on this loophole, and just take the statement that $n$ is even to be part of the best reductionist system, which justifies promoting it to the status of a law, thereby avoiding my main argument. However, first of all, it just seems that such a statement is not at all the kind of statement that is a candidate for being a law - why would it be nomologically necessary that $n$ is even? Secondly, it could well be false of the actual world that $n$ is even. Thirdly, even if $n$ just happens to be even in the actual world, the reductionist still has to prove that $G \mathbf{m}=\mathbf{a}$ is solvable (where $G$ is given by Eq. 9). The attractive nature of gravity is enough to show that even in those worlds there will be initial spatiotemporal conditions that do not correspond to any set of positive ${ }^{34}$ masses, namely those where the particles 'on the outside' have an acceleration that points away from all the other matter. Fourthly, it may seem that adding this extra law makes the axiomatic system 'better' by making it much stronger at the cost of only a small reduction of simplicity, but given the sheer amount of dynamically possible worlds ( $\gg n^{n}$ where $n$ is a large number), merely halving that large number at the cost of moving from four laws to five is actually not that advantageous ${ }^{35}$. Fifthly, assuming a nonrevisionary reductionist - as suggested in Section 2-who wants to reproduce

\footnotetext{
33 Dasgputa [35] is developing an analogue of this project in response to the accusation that relationalism about handedness, space and mass are all indeterministic. That case seems much simpler though than the case considered in this paper.

34 Although, for e.g. a system with four masses on the vertices of a square, all with accelerations of equal magnitude pointing outwards along the diagonals, there is one unique solution if we were to allow negative masses. (It consists of masses of equal magnitude (the exact value depending on the acceleration magnitude), but the masses on one diagonal have a negative sign, whereas the masses on the other diagonal have a positive sign.)

35 I would like to thank David Wallace for pointing this out to me.
} 
all the consequences of and the work being done by the standard form of NG (i.e. with primitive masses), the reductionist theory needs to generate all the empirically possible models of standard NG. This includes models with an odd number of particles, even if none of those represents the actual world. Finally and most importantly, the main argument still goes through for quasi-isolated subsystems of an odd number of particles. Thus, even if our universe consisted of an even number of particles, there will (probably) still be solar systems with an odd number of celestial objects. (It would have been nice for my purposes if that were true of our own solar system -ignoring asteroids etc.--but alas!)

Let us now turn to the last cluster of related issues. The focus in this paper has mainly been on accelerations (and distances). Have we ruled out a reduction of mass to any type or combination of types of spatiotemporal quantities, or only a reduction to accelerations (and distances)? We gain some insight into this question when we return to the issue of operationally defining the absolute mass scale (Subsection 3.1; assuming we would have been able to fix the mass ratios). Earlier I suggested that we could perhaps use the escape velocity scenario for this purpose. The escape velocity inequality obeyed by that scenario can be rewritten in terms of $r, v$ and $a$ only, suggesting that we define the absolute mass scale via some ratio of $r, v$ and $a$. However, Figure 3 has not only proven that mass ratios cannot be reduced to accelerations (and distances), it also proves that the absolute mass scale cannot be defined in terms of $r, v$ and $a$ once we have more than two particles. For in that figure not only the initial distances and accelerations of the two superimposed solutions agreed, but also the initial velocities. Thus, an initial spatiotemporal state containing spatiotemporal quantities up till second order fails to solve the reductionist project.

Could we include higher-order spatiotemporal quantities? Since these cannot be analytically determined from NG with primitive masses, it is difficult to answer this question ${ }^{36}$, but since this would mean adding even more 'degrees of freedom' this does not seem to be a viable option. We are trying to reduce $n$ mass degrees of freedom to more than $n$ spatiotemporal quantities. These extra quantities cannot be truly degrees of freedom; they cannot be independent of the $n$ degrees of freedom. Either they 1) will lead to inconsistencies in the determination of the masses, or 2) they will always conspire to take on exactly

\footnotetext{
36 Perhaps the following serves as a plausibility argument for an upper bound on the order $k$ of initial spatiotemporal data that would guarantee removing the underdetermination of mass (although the overdetermination problem, resulting in conspiratorial (i.e. unexplained!) constraints, still remains). On one popular view, the "at-at" theory of motion, (initial) velocities are not in fact properties of an (initial) instant, but of an infinitesimal (initial) period of time. After all, velocity is usually defined as $\lim _{d t \rightarrow 0} \frac{r(t+d t)-r(t)}{d t}$, which is a property intrinsic to $[t, t+d t]$. In general, the initial $k^{\text {th }}$-order time derivative of $r$ is a property of $\left[t_{0}, t_{0}+k d t\right]$. In a slogan: 'God was not done when he created the initial configuration and the laws, but he had to also specify the subsequent $k-1$ configurations (depending on the order of initial spatiotemporal data that we are considering)'. Now, if $k=n+1$, this initial period (of $n+1$ instants) effectively contains $n-1$ independent sets of accelerations (and even more sets of distances). Narlikar's method then guarantees that this initial data fixes the masses.
} 
the right values as to avoid inconsistencies. Such a mysterious, conspiratorial constraint - which presumably cannot even be formulated without referring to mass $^{37}$ — would be totally unexplained, even if imposing this constraint on the initial spatiotemporal state would uniquely fix the evolution.

\section{Conclusion}

Laplace wondered about the minimal choice of initial variables and parameters corresponding to a well-posed initial value problem. This paper considered Laplace's problem in the context of Newtonian Gravity, and asked whether mass needs to be included in the initial state, or whether it could be substituted with a purely spatiotemporal initial state. It has been argued that such Laplacian reduction of Newtonian mass to spatiotemporal quantitiesespecially accelerations and distances - is false. Removing mass from the initial state drastically reduces the predictive and explanatory power of Newtonian Gravity. As a corrolary, Laplacian reductionism is also false in the context of MOND.

\section{References}

1. H. Poincaré, Science and Hypothesis (Dover, New York, 1952 [1902]). Translated by W. Scott.

2. J. Barbour, The end of time: The next revolution in physics (Oxford: Oxford University Press, 2000)

3. B. Skow, British Journal for the Philosophy of Science 58, 777 (2007)

4. O. Pooley, The reality of spacetime. Dphil thesis, Oriel College, University of Oxford (2002)

5. S. Dasgupta, in Oxford Studies in Metaphysics, vol. 8, ed. by K. Bennett, D.W. Zimmerman (Oxford University Press, 2013), pp. 105-147

6. D.J. Baker, Some consequences of physics for the comparative metaphysics of quantity. Manuscript, May 2013

7. N.C.M. Martens, Against comparativism about mass in newtonian gravity - a case study in the metaphysics of scale. Dphil thesis, Magdalen College, University of Oxford (2017)

8. M. Milgrom, The Astrophysical Journal 270, 365

9. N.C.M. Martens, The (un)detectability of absolute newtonian masses. Manuscript

10. J.S. Bell, Speakable and unspeakable in quantum mechanics (Cambridge University Press, 1987)

11. P.R. Holland, The Quantum Theory of Motion: An Account of the de Broglie-Bohm Causal Interpretation of Quantum Mechanics (Cambridge University Press, 1995)

12. M. Jammer, Concepts of Mass in Contemporary Physics and Philosophy (Princeton University Press, 2000)

13. H. Zanstra, Physical Review 23(4), 528 (1924)

14. N.C.M. Martens, Philosophy of Science 84(5), 1226 (2017)

15. M. Dees, Against quantity primitivism. Manuscript

16. Z. Perry, Chapter 4. problems for a dynamic theory of quantity (2016). Part of PhD thesis manuscript

17. M. Esfeld, D.A. Deckert, A minimalist ontology of the natural world (New York: Routledge, 2017)

37 See fn.30. 
18. E. Mach, The Science of Mechanics (The Open Court Publishing Co., translated by T.J. McCormack, 1960 [1893])

19. C. Pendse, The London, Edinburgh, and Dublin Philosophical Magazine and Journal of Science: Series $7 \mathbf{2 7}(180), 51$ (1939)

20. H.A. Simon, The London, Edinburgh, and Dublin Philosophical Magazine and Journal of Science: Series 7 38(287), 888 (1947)

21. N.C.M. Martens, Machian comparativism about mass. Manuscript

22. J. Earman, A Primer on Determinism (D. Reidel Publishing Company, 1986)

23. J.D. Norton, Philosophers' Imprint 3(4), 1 (2003)

24. S. Dasgupta, Philosophers' Imprint 14(20), 1 (2014)

25. C. Pendse, The London, Edinburgh, and Dublin Philosophical Magazine and Journal of Science: Series 7 24(164), 1012 (1937)

26. V. Narlikar, The London, Edinburgh, and Dublin Philosophical Magazine and Journal of Science: Series 7 27:180, 33 (1939)

27. C. Pendse, The London, Edinburgh, and Dublin Philosophical Magazine and Journal of Science: Series 7 29(196), 477 (1940)

28. E. Knox, British Journal for the Philosophy of Science 65(4), 863 (2014)

29. H.J. Schmidt, Philosophia Naturalis 30, 189 (1993)

30. D. Lewis, Counterfactuals (Cambridge: Harvard University Press, 1973)

31. J. Earman, in Profiles, vol. 4, ed. by D. Armstrong, R. Bogdan (Dordrecht: D. Reidel Publishing Company, 1984)

32. D. Albert, Time and Chance (Cambridge, MA: Harvard University Press, 2000)

33. O. Pooley, The Reality of Spacetime (2016). Manuscript

34. F. Arntzenius, Space, Time, \& Stuff (Oxford University Press, 2012)

35. S. Dasgupta, Relationalism and the limits of possibility. Manuscript, Oct 2015 